Bundesgesundheitsbl 2016 · 59:523-536

DOI 10.1007/s00103-016-2324-9

Online publiziert: 17. März 2016

(c) Springer-Verlag Berlin Heidelberg 2016

CrossMark
Udo Buchholz ${ }^{1}$ Silke Buda' · Annicka Reuß ${ }^{1}$ - Walter Haas' ${ }^{1}$ Helmut Uphoff ${ }^{2}$

${ }^{1}$ Abteilung für Infektionsepidemiologie, Robert Koch-Institut, Berlin, Deutschland

${ }^{2}$ Hessisches Landesuntersuchungs- und Prüfungsamt im Gesundheitswesen, Dillenburg, Deutschland

\title{
Todesfälle durch Influenzapandemien in Deutschland 1918 bis 2009
}

\author{
Schätzwerte auf Basis der Literatur und \\ ergänzende eigene Berechnungen
}

Im 20. und 21. Jahrhundert ereigneten sich 4 Influenzapandemien, deren Beginn global auf die Jahre 1918, 1957, 1968 sowie 2009 datiert wurde. Sie wurden (in chronologischer Reihenfolge) durch die Subtypen A(H1N1), A(H2N2), $\mathrm{A}(\mathrm{H} 3 \mathrm{~N} 2)$ und $\mathrm{A}(\mathrm{H} 1 \mathrm{~N} 1)$ pdm09 verursacht. Problematisch ist die Bezifferung der pandemiebedingten Todesfallanzahl in Deutschland durch die z. T. lückenhafte Datenlage - v. a. für die Pandemie 1918/1919 - und mühsame Erhebung schriftlich dokumentierter Zahlen an verschiedenen Stellen sowie die unterschiedliche Qualität und zeitliche Auflösung der Daten in den verschiedenen Zeitabschnitten. Ein grundsätzliches Problem ist auch, dass die Zahl der Todesfälle, die direkt oder indirekt mit Influenzainfektionen zusammenhängt, generell nicht zuverlässig direkt aus Statistiken der Todesursachen, die von Ärzten in Leichenschauscheinen angegeben werden, herausgelesen werden kann, da „Influenza“ viel zu selten diagnostiziert und/oder angegeben wird [1, 2]. Mit Influenza assoziierte Todesfälle können sich ganz allgemein bei den respiratorischen Todesursachen, aber auch bei Herz-Kreislauf-Erkrankungen und anderen chronischen Vorerkrankungen verbergen $[1,3]$. Wegen der schwierigen Quantifizierung influenzaassoziierter Todesfälle wurde schon in den 1960erJahren von Serfling eine Methode entwickelt, um aus Zeitreihen (z.B. zur Gesamtmortalität) die während der In- fluenzawelle über das übliche Maß (ohne Influenzawelle) hinausgehende Mortalität abschätzen zu können (sog. ExzessMortalität) [4]. In der Folgezeit kamen auch andere Formen von Zeitreihenmodellierungen zur Anwendung [5-8]. Eine weitere Problematik besteht darin, dass sich mehrere Wellen mit unterschiedlicher Intensität ereignen können und keine klaren Kriterien existieren, welche der Wellen man der jeweiligen Pandemie zurechnet. Daher sollte bei der Beschreibung der Auswirkungen einer Pandemie, z. B. ausgedrückt als ExzessMortalität, angegeben werden, auf welche Pandemiewellen sie sich beziehen.

Die weltweite Zahl der Verstorbenen der Pandemie 1918/1919 wurde Ende der 1920er-Jahre auf 21,5 Mio. geschätzt [9] und infolge späterer Nachforschungen auf rund 50 Mio. korrigiert [10]. Die Weltgesundheitsorganisation (WHO) gab im Jahr 2013 für die dPandemien des 20. Jahrhunderts an, dass 1918/1919 20-50 Mio., 1957/1958 1-4 Mio., und 1968/1969 ebenfalls 1-4 Mio. Menschen pandemiebedingt verstorben seien [11]. Für die Pandemie 2009 wurde von zwei unabhängigen Arbeitsgruppen mittels mathematischer Modelle eine Zahl von ca. 200.000 Todesfällen allein aufgrund respiratorischer Erkrankungen geschätzt $[8,12]$.

Wie viele Opfer die genannten Pandemien in Deutschland gefordert haben, ist insbesondere für die Pandemie 1918/1919 weitgehend unbekannt. Darü- ber hinaus ist unseres Wissens die Mortalität aufgrund dieser vier Pandemien in Deutschland noch nicht ausführlich recherchiert und in einer umfassenden Veröffentlichung zusammengestellt worden. In einem ersten Schritt sollte daher eine Literaturrecherche durchgeführt werden, um wissenschaftlich fundierte Angaben zur Zahl der Todesfälle infolge der Pandemien des 20. Jahrhunderts und 2009 in der deutschen Bevölkerung zu identifizieren. $\mathrm{Zu}$ allen Pandemien sollten darüber hinaus eigene Schätzungen, basierend auf ExzessMortalitäts-Berechnungen, erfolgen; bereits veröffentlichte Auswertungen sollten gegebenenfalls ergänzt werden. Die verschiedenen Werte aus der Literatur und den eigenen Berechnungen sollten bezüglich Methodik und Qualität der Datengrundlage bewertet werden, um den jeweils Vertrauenswürdigsten zu identifizieren. Aus den Todesfallzahlen sollte für jede Pandemie eine Letalität berechnet und mit globalen Werten der WHO verglichen werden.

\section{Methodik}

\section{Literatursuche}

- Die Literaturdatenbanken Pubmed und Scopus wurden auf passende Veröffentlichungen zu den Begriffen „Death“, „Mortality“ (als MESH-Begriffe), „Fatal*“, „Impact“, „Burden“ (neben) „disease“, „statistics“ 
- UND „Epidemic*“, „Disease outbreak" (MESH-Begriff; schließt ein: "Epidemics" und "Pandemic")

- UND „Influenza“ (MESH-Begriff), „Grippe“, „Spanish flu“, „Hong Kong flu“, „Asian flu“, „Influenza pandemic, 1918-1919“ (Mesh-Begriff), UND „German*“", „deutsch“, „Kaiserreich“, „Reich“

- UND „1918“, „1957“, „1968“, „2009“ durchsucht. Zusätzlich wurde im eigenen Literaturfundus nach relevanter Literatur gesucht. Außerdem wurde das statistische Bundesamt bezüglich historischer Todesfallsowie Bevölkerungszahlen, die auch den Zeitraum der ersten Pandemie 1918/1919 umfassen sollten, kontaktiert.

\section{Begriffserläuterung}

Exzess-Mortalitäts-Schätzungen für eine (pandemische) Influenzawelle basieren prinzipiell darauf, dass die Mortalität während der Influenzawelle gegenüber dem „zu erwartenden“ Hintergrundgeschehen subtrahiert und der Influenzawelle zugeschrieben wird. Bei dem „zu erwartenden“ Hintergrundgeschehen kann eine „übliche“ Influenzawelle einbezogen werden oder auch nicht. Das bedeutet, dass, wenn eine Hintergrundmortalität mit üblicher Influenzawelle $\mathrm{zu}$ Grunde gelegt wird, die geschätzte Exzess-Mortalität geringer ausfällt, als wenn die Mortalität ohne übliche Influenzawelle den Hintergrund bildet. In dieser Arbeit wird dann, wenn die Werte von Exzess-Mortalitäts-Schätzungen angegeben werden, in Klammern durch Hinzufügen von „mit Influenza im Hintergrund“ oder „ohne Influenza im Hintergrund" präzisiert, um welche Art der Schätzung es sich handelt.

\section{Eigene Berechnungen bzw. Schätzungen}

Für die Pandemie 1918/1919 wurden uns seitens des statistischen Bundesamts jährliche Mortalitätsdaten einer Auswahl registrierter Todesfälle sowie der Gesamtzahl aller Todesfälle für den Zeitraum von 1892-1971 zur Verfügung gestellt. Monatliche oder wöchentliche
Daten standen uns zu dieser Pandemie nicht zur Verfügung. Eine einfache Schätzung der Exzess-Todesfälle (mit Influenza im Hintergrund) wurde so durchgeführt, dass die Zahl der Gesamttodesfälle 1917 (vorletztes Kriegsjahr) und 1919 (1. Nachkriegsjahr) gemittelt und von der Zahl im Jahr 1918 subtrahiert wurde.

Für eine Schätzung der Todesfälle in den Pandemien 1957/1958 und 1968-1970 lag eine Datenreihe des statistischen Bundesamts zur Anzahl der monatlich in Deutschland verstorbenen Personen von $1946 / 1947$ bis $1999 / 2000$ vor [13]. Aufgrund der zusätzlich vorliegenden Informationen über virologisch bestätigte Influenzaerkrankungen war bekannt, in welchen Monaten Influenzaviren zirkulierten. Für die Modellierung der Hintergrundmortalität (ohne Monate mit Influenzazirkulation) wurde eine Methode verwendet (Modell der relativen Mortalitätsverteilung), die ausführlich in einer Veröffentlichung von Zucs et al. beschrieben wurde [1]. Kurz zusammengefasst wurde dabei über alle Monate ohne Influenzazirkulation der Anteil der Mortalität pro Monat relativ zur jeweiligen Jahresmortalität ermittelt. Die „erwartete“ Mortalität (ohne Influenza im Hintergrund) wurde so berechnet, dass die monatlichen Anteile mit der jährlichen Gesamtmortalität multipliziert wurden. Um die Übersterblichkeit in den Monaten mit Influenzazirkulation zu schätzen, wurde die erwartete Mortalität von der tatsächlich aufgetretenen Mortalität subtrahiert. Für die hier vorliegende Arbeit wurde zusätzlich die geschätzte Mortalität für die Bevölkerung der damaligen Bundesrepublik Deutschland (BRD) und Deutschen Demokratischen Republik (DDR) auch in die Zahl der ExzessTodesfälle umgerechnet.

Für die Pandemie 2009 gab es drei bereits publizierte Arbeiten, die die Autoren (mit-)verfasst hatten. Auch wenn für die vorliegende Veröffentlichung keine zusätzlichen Berechnungen durchgeführt wurden, sollen sie der Vollständigkeit halber an dieser Stelle ebenfalls kurz beschrieben werden, v. a., weil die zweite Arbeit in der publizierten Form die Details für die Auswertung in Deutschland nicht beschreibt: In der ersten Arbeit wurde die Zahl der Personen ermittelt, die aufgrund einer Infektion mit dem pandemischen Virus A(H1N1)pdm09 verstorben waren, an die Gesundheitsämter gemeldet und über die Landesstellen an das Robert Koch-Institut (RKI) übermittelt worden waren [14].

In der zweiten Arbeit [8] standen aggregierte Zahlen von Verstorbenen ab 1998 zur Verfügung, die vom Forschungdatenzentrum des Bundes und der Länder erhältlich waren (www.forschungsdatenzentrum. de/). Die Zeitreihe endete jedoch am 31.12.2009. Aggregiert für Altersgruppe, Todeswoche und Todesursache lagen für das Alter bzw. die zugrunde liegende Todesursache folgende Daten vor: (1) Alter: Zahl der Verstorbenen (a) ohne Berücksichtigung der Altersgruppen und (b) stratifiziert nach den Altersgruppen „unter 65 Jahre“ bzw. „65 Jahre und älter"; (2) Todesursache: (a) ohne Berücksichtigung der Todesursache, i.e. die gesamte Zahl der Sterbefälle (Gesamtmortalität); (b) Beschränkung auf die Verstorbenen mit Todesursache einer der ICD-Codes J09-J18 (Grippe oder Pneumonie); (c) Beschränkung auf die Verstorbenen mit jeglicher respiratorischen Todesursache (J00-J99) und (d) Beschränkung auf die Verstorbenen mit jeglicher respiratorischen (J00-J99) oder kardiovaskulären Todesursache (I00-199). Für die verschiedenen AltersTodesursachen-Kombinationen wurden in den jeweiligen Todesfallkategorien bzw. Altersgruppen multivariate lineare Regressionsmodelle erstellt, wobei als unabhängige Größen Daten aus der virologischen Surveillance sowie zeitliche Trends verwendet wurden. Die Modelle schätzten (ohne Influenza im Hintergrund) die Zahl der infolge einer Infektion durch das pandemische Virus A(H1N1)pdm09 verstorbenen Personen.

In der dritten Arbeit [15] standen auch Daten aus dem Jahre 2010 zur Verfügung, jedoch nur auf monatlicher Basis, ohne Altersgruppenangabe und ohne Information zur Todesursache. Das Schätzverfahren war das bereits genannte nach Zucs et al. [1]. 
Bundesgesundheitsbl 2016 · 59:523-536

(c) Springer-Verlag Berlin Heidelberg 2016

U. Buchholz · S. Buda · A. Reuß - W. Haas · H. Uphoff

\section{Todesfälle durch Influenzapandemien in Deutschland 1918 bis 2009. Schätzwerte auf Basis der Literatur und ergänzende eigene Berechnungen}

\section{Zusammenfassung}

Hintergrund und Zielstellung. Schätzungen der Anzahl der Verstorbenen infolge der Influenzapandemien des 20. und 21. Jahrhunderts $(1918 / 1919,1957 / 1958,1968-1970$ und 2009) sind weltweit und auch für Deutschland eine Herausforderung. Die aufgrund einer systematischen Literaturrecherche erhobenen Ergebnisse sollen zusammengetragen, durch eigene Berechnungen ergänzt und bewertet werden.

Methoden. Es wurde eine systematische Literaturrecherche unter Verwendung u. a. der Begriffe death, mortality, pandemic, epidemic, Germany, 1918, 1957, 1968, 2009 durchgeführt. Treffer wurden nach Titel bzw. Zusammenfassung gesichtet und bezüglich möglicher Relevanz selektiert. Eigene Schätzungen wurden, basierend auf ExzessMortalitäts-Berechnungen, durchgeführt. Die verschiedenen Werte aus der Literatur und den gegebenenfalls eigenen Berechnungen wurden bezüglich Methodik und Qualität der
Datengrundlage bewertet, um den jeweils vertrauenswürdigsten zu identifizieren. Aus den Todesfallzahlen wurde für jede Pandemie eine Letalität berechnet und mit globalen Werten der Weltgesundheitsorganisation (WHO) verglichen.

Ergebnisse. Für die Pandemie 1918/1919 identifizierten wir 5 , für die Pandemien 1957/1958 und/oder 1968-1970 3 und für die Pandemie 2009 ebenfalls 3 relevante Publikationen. Für alle 4 Pandemien waren die plausibelsten Schätzungen diejenigen, welche mittels einer Zeitreihenanalyse (aus der Literatur entnommen oder aufgrund eigener Schätzungen) monatlicher oder wöchentlicher Gesamttodesfallstatistiken ermittelt wurden. Diese waren für die 4 Pandemien in chronologischer Reihenfolge 426.600 (1918/1919), 29.100 (1957/1958), 46.900 (1968-1970) bzw. 350 (2009) und entsprachen einer bevölkerungsbezogenen Exzess-Mortalität zwischen 691/100.000
(0,69 \%) infolge der Pandemie 1918/1919 und $0,43 / 100.000(0,00043 \%)$ für 2009. Die resultierenden Letalitäten stimmten gut mit globalen, von der WHO angegebenen Werten überein.

Schlussfolgerungen. Für die letzten 4 Pandemien (1918 bis 2009) konnten plausible Schätzungen der Exzess-Todesfallzahlen identifiziert werden. Die Heterogenität der entsprechenden Exzess-Mortalität zwischen den Pandemien ist bei einem Faktor von mehr als 1000 enorm. Diese kann durch Eigenschaften des Virus oder des Wirts (Immunität), die jeweiligen gesellschaftlichen Bedingungen, die Gesundheitsversorgung oder die medizinische Entwicklung bedingt sein.

Schlüsselwörter Pandemie · Exzess-Mortalitäts-Schätzung · Influenza · Grippe · Mortalität

\section{Influenza pandemic deaths in Germany from 1918 to 2009. Estimates based on literature and own calculations}

\section{Abstract}

Background and objective. Estimation of the number of deaths as a consequence of the influenza pandemics in the twentieth and twenty-first centuries (i.e. 1918-1919, 1957-1958, 1968-1970 and 2009) is a challenge worldwide and also in Germany. After conducting a systematic literature search complemented by our own calculations, values and estimates for all four pandemics were collated and evaluated.

Method. A systematic literature search including the terms death, mortality, pandemic, epidemic, Germany, 1918, 1957, 1968,2009 was performed. Hits were reviewed by title and abstract and selected for possible relevance. We derived our own estimates using excess mortality calculations, which estimate the mortality exceeding that to be expected. All identified values were evaluated by methodology and quality of the database. Numbers of pandemic deaths were used to calculate case fatality rates and were compared with global values provided by the World Health Organization.

Results. For the pandemic 1918-1919 we identified 5 relevant publications, 3 for the pandemics 1957-1958 and 1968-1970 and 3 for 2009. For all four pandemics the most plausible estimations were based on time series analyses, taken either from the literature or from our own calculations based on monthly or weekly all cause death statistics. For the four pandemics these estimates were in chronological order 426,600 (1918-1919), 29,100 (1957-1958), 46,900 (1968-1970) and 350 (2009) excess pandemic-related deaths. This translates to an excess mortality ranging between 691 per 100,000 (0.69\% in
1918-1919) and 0.43 per 100,000 (0.00043\% in 2009). Case fatality rates showed good agreement with global estimates. Conclusion. We have proposed plausible estimates of pandemic-related excess number of deaths for the last four pandemics as well as excess mortality in Germany. The heterogeneity among pandemics is large with a variation factor of more than 1000 . Possible explanations include characteristics of the virus or host (immunity), social conditions, status of the healthcare system and medical advances.

Keywords Pandemic - Excess mortality estimation . Influenza - Grippe · Mortality 


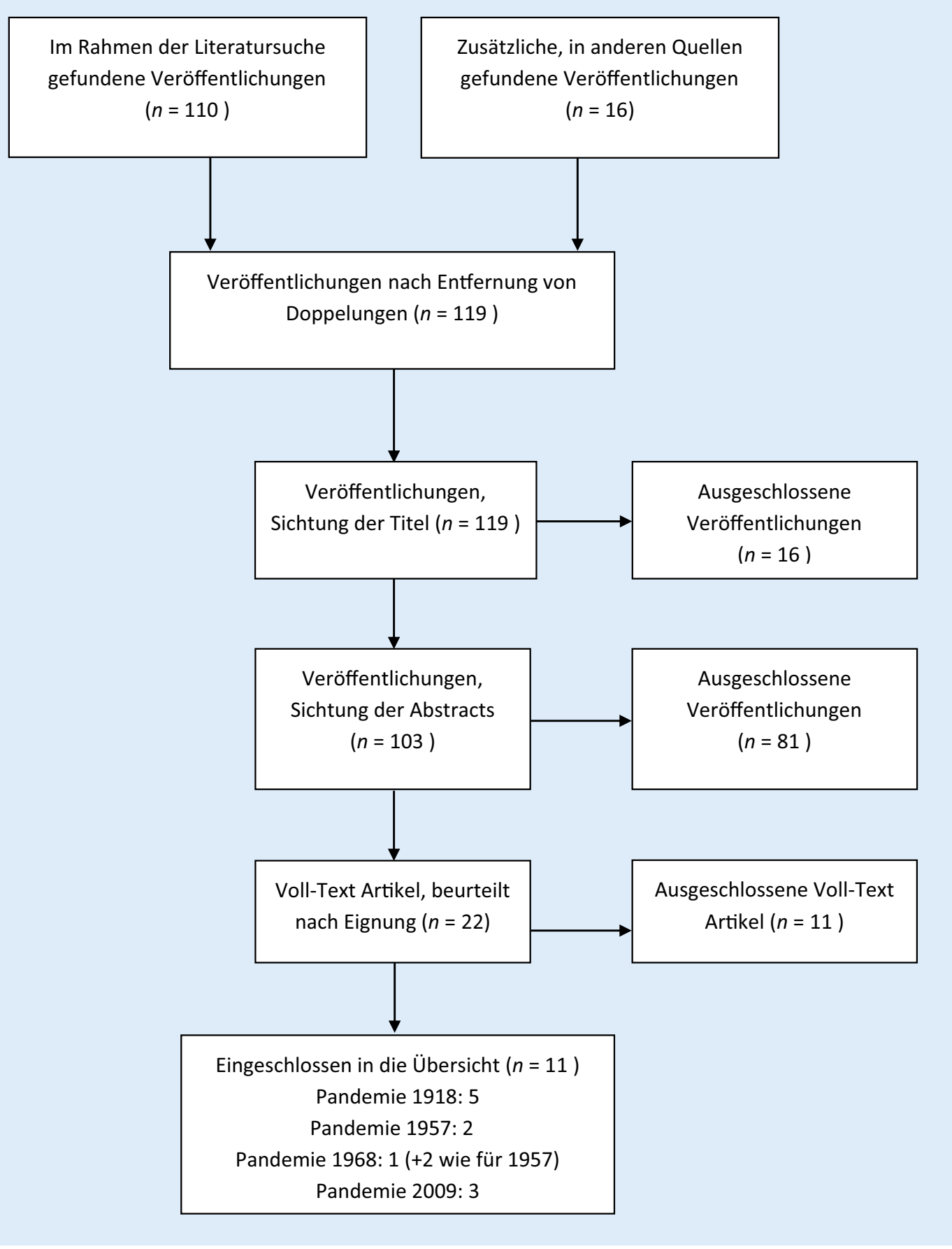

Abb. $1<$ PRISMA-FlowDiagramm zur Zahl der im Rahmen der Suchstrategie und durch andere Quellen gefundenen Veröffentlichungen und schrittweise Einengung bis zur Auswahl der in die Übersicht eingeschlossenen Veröffentlichungen

\section{Bewertungsverfahren der identifizierten Werte}

Um im Rahmen dieser Arbeit die verschiedenen Schätzungen bewerten und eine Auswahl für den plausibelsten Schätzer treffen zu können, wurden die Methode der Auswertung und die zeitliche Auflösung der Datengrundlage herangezogen. Bei den verwendeten Auswertemethoden gibt es prinzipiell schät- zende Methoden und „registrierende“ Methoden. Als registrierende Methoden werden diejenigen bezeichnet, die ausschließlich als „Influenza“ registrierte Todesfälle, die während einer Pandemie registriert wurden, addieren. Registrierende Methoden beziffern naturgemäß die pandemiebezogene Mortalität ohne Influenza im Hintergrund. Im Allgemeinen werden schätzende Methoden den registrierenden Methoden vorgezogen.
Bei den schätzenden Methoden werden alle Todesfälle oder die Todesfälle bestimmter Todesursachenkategorien verwendet (z. B. „Pneumonie“), um damit einen während einer pandemischen Erkrankungswelle oder Erkrankungswellen zu beobachtenden Exzess zu berechnen (je nach Methode mit bzw. ohne Influenza im Hintergrund). Innerhalb der Schätzverfahren werden modellierende Zeitreihenanalysen einfa- 
Tab. 1 Veröffentlichungen (Literaturfunde) bzw. eigene Berechnungen der Autoren zur Schätzung der Todesfälle in Deutschland aufgrund der Pan-

demien 1918/1919, 1957/1958, 1968-1970 und 2009

\begin{tabular}{|c|c|c|c|c|c|c|c|c|}
\hline $\begin{array}{l}\text { Literatur- } \\
\text { stelle oder } \\
\text { Eigene Be- } \\
\text { rechnung }\end{array}$ & $\begin{array}{l}\text { Erstautor } \\
\text { (Publika- } \\
\text { tion) }\end{array}$ & $\begin{array}{l}\text { Publikations- } \\
\text { datum }\end{array}$ & Datengrundlage & Methodik & $\begin{array}{l}\text { Bevölkerung } \\
\text { in Deutsch- } \\
\text { land } \\
\text { während } \\
\text { Pandemie }\end{array}$ & $\begin{array}{l}\text { Exzess- } \\
\text { Mortalität } \\
\text { pro } \\
100.000 \\
\text { Bevöl- } \\
\text { kerung }\end{array}$ & $\begin{array}{l}\text { Geschätzte } \\
\text { Zahl der } \\
\text { Exzess- } \\
\text { Todesfälle, } \\
\text { gerundet }\end{array}$ & Bemerkung \\
\hline \multicolumn{9}{|c|}{ Pandemie 1918/1919 (A[H1N1]; „Spanische Grippe“) } \\
\hline Literatur & $\begin{array}{l}\text { Patterson } \\
\text { [19] }\end{array}$ & 1991 & Todesfallstatistik? & $\begin{array}{l}\text { Unklar, vermutlich } \\
\text { keine Modellierung }\end{array}$ & 61.756 .930 & 445 & $\begin{array}{l}250.000 \\
300.000\end{array}$ & $\begin{array}{l}\text { 2. (Herbst-) } \\
\text { Welle } 1918\end{array}$ \\
\hline Literatur & Johnson [10] & 2002 & Todesfallstatistik? & $\begin{array}{l}\text { Für Deutschland } \\
\text { nicht klar }\end{array}$ & 61.756 .930 & 365 & 225.300 & $\begin{array}{l}\text { 1.-4. Welle, i.E. } \\
\text { 1918-1920 }\end{array}$ \\
\hline Literatur & Michels [20] & 2010 & $\begin{array}{l}\text { Jährliche Todesfall- } \\
\text { statistik „Grippe” } \\
\text { oder „Pneumonie“ }\end{array}$ & $\begin{array}{l}\text { "Exzess-Berechnung" } \\
\text { (Vergleich } 1918 \text { mit } \\
\text { 1917) }\end{array}$ & 61.756 .930 & 389 & 240.000 & $\begin{array}{l}\text { 1. }+2 . \text { Welle } \\
\text { 1918; von } \\
\text { Mecklenburg } \\
\text { keine Daten } \\
\text { vorhanden }\end{array}$ \\
\hline Literatur & Michels [20] & 2010 & $\begin{array}{l}\text { Jährliche Todesfall- } \\
\text { statistik „Grippe“ } \\
\text { oder „Pneumonie“ }\end{array}$ & $\begin{array}{l}\text { "Exzess-Berechnung" } \\
\text { (Vergleich } 1918 \\
\text { mit 1917) }+3 . \\
\text { Welle 1919+ Tote } \\
\text { durch Encephalitis } \\
\text { lethargica }\end{array}$ & 61.756 .930 & 542 & $\begin{array}{l}320.000- \\
350.000\end{array}$ & $\begin{array}{l}\text { 1.-3. Welle } \\
\text { (1918 und } \\
\text { 1919), inklu- } \\
\text { sive Todesfälle } \\
\text { durch En- } \\
\text { cephalitis } \\
\text { lethargica }\end{array}$ \\
\hline Literatur & Murray [21] & 2006 & $\begin{array}{l}\text { Jährliche Statistik } \\
\text { aller Todesfälle } \\
\text { ohne Berücksichti- } \\
\text { gung der Ursache }\end{array}$ & $\begin{array}{l}\text { Einfache Modell- } \\
\text { rechnung (Summe } \\
\text { der Differenz der } \\
\text { jährlichen Morta- } \\
\text { lität (1918, 1919, } \\
\text { 1920) zum Dur- } \\
\text { chschnittswert } \\
\text { (1921-1923) nach } \\
\text { der Pandemie) }\end{array}$ & 61.756 .930 & 760 & 469.400 & $\begin{array}{l}\text { 1., 2., 3. und } \\
\text { 4. Welle }\end{array}$ \\
\hline Literatur & Ansart [22] & 2009 & $\begin{array}{l}\text { Monatliche Statistik } \\
\text { aller Todesfälle } \\
\text { 1906-1936 ohne } \\
\text { Berücksichtigung } \\
\text { der Ursache }\end{array}$ & $\begin{array}{l}\text { Periodisches Re- } \\
\text { gressionsmo- } \\
\text { dell für 1. und } \\
\text { 2. Welle (1918), } \\
\text { basierend auf mo- } \\
\text { natlichen Todesfall- } \\
\text { daten (1906-1936) }\end{array}$ & 61.756 .930 & 691 & 426.600 & $\begin{array}{l}\text { 1. und } \\
\text { 2. Welle, beide } \\
\text { in } 1918\end{array}$ \\
\hline $\begin{array}{l}\text { Eigene Be- } \\
\text { rechnung }\end{array}$ & $\begin{array}{l}\text { Buchholz } \\
\text { (dieses Ma- } \\
\text { nuskript) }\end{array}$ & 2016 & $\begin{array}{l}\text { Jährliche Statistik } \\
\text { aller Todesfälle } \\
\text { ohne Berücksichti- } \\
\text { gung der Ursache }\end{array}$ & $\begin{array}{l}\text { Differenz zwischen } \\
1918 \text { und Mittel aus } \\
1917 \text { und } 1919\end{array}$ & 61.756 .930 & 716 & 442.300 & $\begin{array}{l}\text { 1. und 2. Welle } \\
\text { in } 1918\end{array}$ \\
\hline \multicolumn{9}{|c|}{ Pandemie 1957/1958 (A[H2N2]; „Asiatische Grippe“) } \\
\hline Literatur & Meyer [24] & 1974 & $\begin{array}{l}\text { Sterbetafeln } \\
1950-1973\end{array}$ & $\begin{array}{l}\text { Zeitreihenanalyse } \\
\text { der Gesamtto- } \\
\text { desfälle }\end{array}$ & 72.030 .866 & 26 & $\begin{array}{l}17.200- \\
19.800\end{array}$ & $\begin{array}{l}\text { Exzess nur für } \\
\text { Oktober } 1957 \\
\text { berechnet; } \\
\text { extrapoliert } \\
\text { für BRD + DDR }\end{array}$ \\
\hline $\begin{array}{l}\text { Eigene Be- } \\
\text { rechnung }\end{array}$ & Uphoff [13] & 2004 & $\begin{array}{l}\text { Monatliche Statistik } \\
\text { aller Todesfälle } \\
\text { 1947-2000 ohne } \\
\text { Berücksichtigung } \\
\text { der Ursache }\end{array}$ & $\begin{array}{l}\text { Modellrechnung } \\
\text { basierend auf mo- } \\
\text { natlichem Morta- } \\
\text { litätsmuster }\end{array}$ & 72.030 .866 & 40 & 29.100 & $\begin{array}{l}\text { September } \\
1957+\text { Ok- } \\
\text { tober } 1957 \\
\text { sowie Januar } \\
1958+ \\
\text { Februar 1958; } \\
\text { BRD + DDR }\end{array}$ \\
\hline
\end{tabular}


Tab. 1 Veröffentlichungen (Literaturfunde) bzw. eigene Berechnungen der Autoren zur Schätzung der Todesfälle in Deutschland aufgrund der Pandemien 1918/1919, 1957/1958, 1968-1970 und 2009 (Fortsetzung)

\begin{tabular}{|c|c|c|c|c|c|c|c|c|}
\hline $\begin{array}{l}\text { Literatur- } \\
\text { stelle oder } \\
\text { Eigene Be- } \\
\text { rechnung }\end{array}$ & $\begin{array}{l}\text { Erstautor } \\
\text { (Publika- } \\
\text { tion) }\end{array}$ & $\begin{array}{l}\text { Publikations- } \\
\text { datum }\end{array}$ & Datengrundlage & Methodik & $\begin{array}{l}\text { Bevölkerung } \\
\text { in Deutsch- } \\
\text { land } \\
\text { während } \\
\text { Pandemie }\end{array}$ & $\begin{array}{l}\text { Exzess- } \\
\text { Mortalität } \\
\text { pro } \\
100.000 \\
\text { Bevöl- } \\
\text { kerung }\end{array}$ & $\begin{array}{l}\text { Geschätzte } \\
\text { Zahl der } \\
\text { Exzess- } \\
\text { Todesfälle, } \\
\text { gerundet }\end{array}$ & Bemerkung \\
\hline \multicolumn{9}{|c|}{ Pandemie 1968-1970 (A[H3N2]; „Hong Kong Grippe “) } \\
\hline Literatur & Meyer [24] & 1974 & $\begin{array}{l}\text { Sterbetafeln } \\
\text { 1950-1973 }\end{array}$ & $\begin{array}{l}\text { Zeitreihenanalyse } \\
\text { der Gesamtto- } \\
\text { desfälle }\end{array}$ & 78.069 .482 & 66 & 51.200 & $\begin{array}{l}\text { Exzess be- } \\
\text { rechnet für } \\
\text { Dezember } \\
\text { 1969 + Januar } \\
\text { 1970; extrapo- } \\
\text { liert für BRD + } \\
\text { DDR }\end{array}$ \\
\hline Literatur & Casper [25] & 1996 & $\begin{array}{l}\text { Sterbefallstatistik } \\
\text { nach Todesursache } \\
\text { (ICD 9: Code 487) }\end{array}$ & $\begin{array}{l}\text { Nennung der Zah- } \\
\text { len; Addition }\end{array}$ & 78.069 .482 & 20 & 15.300 & $\begin{array}{l}\text { Addition der } \\
\text { Todesfälle, } \\
\text { die als „487“ } \\
\text { (Grippe) ko- } \\
\text { diert waren, } \\
\text { für } 1969 \text { und } \\
1970 \text { (BRD + } \\
\text { DDR) }\end{array}$ \\
\hline $\begin{array}{l}\text { Eigene Be- } \\
\text { rechnung }\end{array}$ & Uphoff [13] & 2004 & $\begin{array}{l}\text { Monatliche Statistik } \\
\text { aller Todesfälle } \\
\text { 1947-2000 ohne } \\
\text { Berücksichtigung } \\
\text { der Ursache }\end{array}$ & $\begin{array}{l}\text { Modellrechnung } \\
\text { basierend auf mo- } \\
\text { natlichem Morta- } \\
\text { litätsmuster }\end{array}$ & 78.069 .482 & 60 & 46.900 & $\begin{array}{l}\text { Dezember } \\
\text { 1969 + Januar } \\
\text { 1970; BRD + } \\
\text { DDR }\end{array}$ \\
\hline \multicolumn{9}{|c|}{ Pandemie 2009 (A[H1N1]pdm09) } \\
\hline Literatur & Wilking [14] & 2010 & $\begin{array}{l}\text { Meldedaten nach } \\
\text { Infektionsschutzge- } \\
\text { setz }\end{array}$ & $\begin{array}{l}\text { Auswertung der } \\
\text { Meldedaten }\end{array}$ & 81.802 .257 & 0,31 & 250 & $\begin{array}{l}\text { September } \\
2009 \text { bis März } \\
2010\end{array}$ \\
\hline Literatur & Simonsen [8] & 2013 & $\begin{array}{l}\text { Todesursachen- } \\
\text { statistik der } \\
\text { Bundesländer } \\
\text { 1998-31.12.2009 }\end{array}$ & $\begin{array}{l}\text { Multivariates line- } \\
\text { ares Regressions- } \\
\text { modell; Model- } \\
\text { lierung der Ge- } \\
\text { samt-Sterbefälle } \\
\text { bei den 0- bis 64-Jä- } \\
\text { hrigen; keine Über- } \\
\text { sterblichkeit bei } \\
\text { den ab 65-Jäh- } \\
\text { rigen }\end{array}$ & 81.802 .257 & 0,43 & 350 & $\begin{array}{l}\text { Verstorbene } \\
\text { bis zum } \\
31.12 .2009\end{array}$ \\
\hline Literatur & RKI [15] & 2011 & $\begin{array}{l}\text { Monatliche Statistik } \\
\text { aller Todesfälle } \\
\text { 1985-2010 ohne } \\
\text { Berücksichtigung } \\
\text { der Ursache }\end{array}$ & $\begin{array}{l}\text { Modellrechnung } \\
\text { basierend auf mo- } \\
\text { natlichem Morta- } \\
\text { litätsmuster }\end{array}$ & 81.802 .257 & 0,00 & 0 & $\begin{array}{l}\text { Epidemiologisch } \\
\text { erhöhte Influ- } \\
\text { enzaaktivität } \\
\text { von Oktober } \\
2009 \text { bis } \\
\text { Januar } 2010\end{array}$ \\
\hline
\end{tabular}

ªevölkerung während der Pandemie 1918 gemittelt aus 1917 und 1919; während der Pandemie 1957/1958 aus Januar 1958; während der Pandemie 1968-1970 aus Januar 1970; während der Pandemie 2009 vom 31.12.2009

chen differenzbildenden Verfahren (z. B. Gesamttodesfälle im Pandemiejahr minus dem Mittelwert aus dem Vor- und Nachpandemiejahr) vorgezogen. Bei der Datengrundlage wurden innerhalb der schätzenden Verfahren Daten mit höherer zeitlicher Auflösung priorisiert, d. h. z. B. wöchentliche Daten höher be- wertet als monatliche Daten, und diese wiederum höher als Jahresdaten.

Synopse der Todesfall- und Mortalitätsdaten aus der Literatur und eigenen Berechnungen

Die als am vertrauenswürdigsten bewerteten Zahlen der Todesfälle bzw. Mor- talität wurden in einer Abbildung gemeinsam mit den Zahlen aus allen Veröffentlichungen bzw. Berechnungen, die in dieser Arbeit genannt wurden, graphisch dargestellt. 
Vergleich der Letalität in Deutschland mit globalen Schätzungen der Weltgesundheitsorganisation

Zusätzlich sollten die Ergebnisse auch mit globalen Angaben der WHO verglichen werden. Da die genannte Quelle der WHO [11] zwar keine Mortalität, dafür aber eine geschätzte Letalität angibt, musste für die für Deutschland identifizierten, plausibelsten Werte erst eine Letalität abgeleitet werden. Dies geschah nach folgender Formel:

\section{Letalität$$
=\frac{\text { Zahl der Todesfälle }}{\text { Erkrankungsrate } \times \text { Bevölkerung }}
$$

Die Zahl der Todesfälle und die Bevölkerungsgröße lagen vor, nur die Erkrankungsrate war nicht bekannt. Daher wurde auf eine Veröffentlichung von Gani zurückgegriffen, in der die klinischen Erkrankungsraten der drei Pandemien $1918 / 1919,1957 / 1958$ und 1968-1970 im Vereinigten Königreich auf 25\%, $31 \%$ bzw. $21 \%$ beziffert wurden und mangels anderer Daten als identisch für Deutschland angenommen wurde [16]. Für die Pandemie im Jahre 2009 wurde die Zahl der Erkrankungen aus deutschen Sentineldaten geschätzt. Von der Arbeitsgemeinschaft Influenza wurde angegeben, dass in der Pandemie 2009 gerundet 3 Mio. Personen aufgrund von Influenzainfektionen einen Arzt konsultierten [17]. Wenn etwa $40 \%$ der Personen mit grippeähnlicher Erkrankung ärztlichen Rat einholten [18], würde danach die Erkrankungsrate in Deutschland ca. $9 \%$ betragen (3 Mio./82 Mio. $\times[1 / 0,4])$.

\section{Ergebnisse}

\section{Literatursuche}

Initial wurden durch die Suchstrategie 110 Veröffentlichungen identifiziert, hinzu kamen weitere 16 Veröffentlichungen aus anderen Quellen (•Abb. 1). Nach schrittweiser Reduktion der Anzahl um die nicht die Einschlusskriterien erfüllenden bzw. thematisch unpassenden Veröffentlichungen verblieben zuletzt 11 Refe- renzen für die Übersicht. Die identifizierten Literaturstellen werden in der Abfolge der einzelnen Pandemien besprochen.

\section{Pandemie 1918/1919}

Identifizierte Literatur: Fünf Veröffentlichungen von Autoren wurden identifiziert, die aufgrund eigener Bemühungen versucht hatten, entweder Originaldaten zu identifizieren, oder Originaldaten verwendeten, um die Zahl der Pandemietodesfälle $1918 \mathrm{zu}$ schätzen (• Tab. 1; [10, 19-22]). Der Historiker K. David Patterson veröffentlichte 1991 einen Artikel zur Geographie und Mortalität der Influenzapandemie 1918 [19]. Darin beschrieb er nicht nur die vermutliche Ausbreitung der ersten und zweiten Welle der Pandemie über die Kontinente und Länder der Erde, sondern widmete sich auch den demographischen Konsequenzen. Die Methodik, wie die Gesamtzahlen zustande kommen, ist ungenau beschrieben. Vermutlich hatte sich der Autor im Sinne der Verwendung einer registrierenden Methode auf diejenigen Todesfälle beschränkt, die als Influenza eingestuft waren; die mangelnde Sensitivität der Erfassung wurde von ihm eingehend thematisiert. In dem Artikel wird für Deutschland eine Gesamtzahl von 250.000-300.000 Pandemietodesfällen geschätzt (gemittelte Mortalität: 445 pro 100.000; • Tab. 1).

Johnson u. Mueller unternahmen 2002 im Bulletin of the History of Medicine einen Versuch, die globalen Auswirkungen der Pandemie im Jahr 1918 auf die Mortalität neu zu taxieren [10]. Dabei wurden sowohl die Originalstatistiken noch einmal überprüft, teilweise auch (einfache) Exzess-Berechnungen berücksichtigt, oder regionale Daten auf die jeweilige nationale Ebene extrapoliert. Welche Methode für die einzelnen Länder durchgeführt wurde, wurde auch für Deutschland - nur unvollständig dokumentiert. Obwohl die weltweite Gesamtzahl der Todesfälle, die in der Veröffentlichung von Patterson noch mit 24,7-39,3 Mio. angegeben worden war, nun auf 50 Mio. hochgestuft worden war („vielleicht sogar zu 100 \% unterschätzt“), wurde die Zahl der Pandemietodesfälle für Deutschland mit 225.330 Todesfällen
(Mortalität: 365/100.000) präzisiert und damit etwas niedriger veranschlagt als bei Patterson (• Tab. 1).

Im Jahr 2010 veröffentlichte der Neuzeithistoriker Michels einen Aufsatz zum Verlauf und den Folgen der Spanischen Grippe im Kontext des 1. Weltkriegs, in dem auch die Zahl der Verstorbenen durch die Pandemie 1918 thematisiert wurde [20]. Die große Zahl der Verluste des Deutschen Reichs im 1. Weltkrieg von 1914-1918 ( $n=2.997 .700)$ [23] erschwerte die Schätzung selbst eines so einschneidenden Ereignisses, wie das der Pandemie 1918. Michels zitiert die Berechnungen des damaligen Reichsgesundheitsamts, wonach die Zahl der gemäß Todesursachenstatistik aufgrund von Grippe oder Lungenentzündung im Jahr 1918 verstorbenen Personen (Zivilisten bzw. Soldaten) mit der Zahl in 1917 verglichen wurde - quasi die einfachste Form einer Exzess-Mortalitäts-Berechnung (Subtraktion zweier Jahre; mit Influenza im Hintergrund). Während im Jahr 1918 bei 340.000 Verstorbenen Grippe oder Lungenentzündung als Todesursache angegeben war (316.000 Zivilisten, 24.000 Soldaten), war dies im Jahr 1917 nur bei 101.800 der Fall, woraus sich eine Zahl von etwa 240.000 Todesfällen (Mortalität 389/100.000) ergab, ähnlich dem Wert von Johnson u. Mueller (• Tab. 1).

In einer weiteren Rechnung wurde die Zahl der Todesfälle in der dritten pandemischen Welle (Anfang 1919) sowie die Zahl der Patienten addiert, die an den Spätfolgen der (der Influenza zugeschriebenen) Encephalitis lethargica verstarben. Die Summe dieser drei Größen (Exzess-Tote in 1918 plus ExzessTote in 1919 plus Verstorbene aufgrund von Encephalitis lethargica) wurde auf 320.000-350.000 (gemittelte Mortalität $542 / 100.000)$ beziffert.

Eine weitere globale Schätzung erfolgte 2006 von Murray et al. [21]. Für die Berechnung der globalen Mortalität wurden nationale, jährliche Mortalitätsdaten herangezogen, die außerhalb der Influenzajahre 1918-1920 (Pandemiezeitraum) aufgetretene Mortalität von 1915-1917 bzw. 1921-1923 gemittelt (mit Influenza im Hintergrund) und dann die jeweilige Differenz aufsummiert. Bei Ländern mit erheblichem 


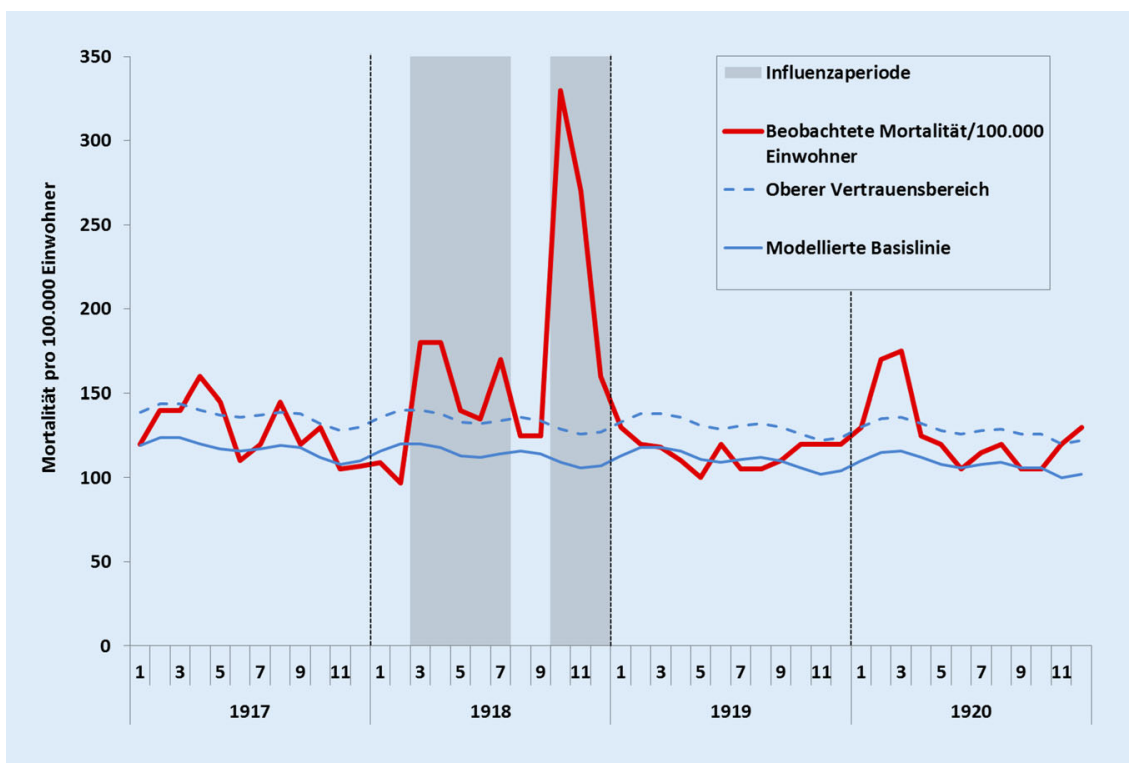

Abb. 2 A Monatliche Gesamtsterberate (pro 100.000 Einwohner) von 1917-1921 in Deutschland. Rote Linie beobachtete Gesamtmortalität pro 100.000 Einwohner; graue Flächen Intervalle der 1. und 2. pandemischen Welle; blaue, durchgezogene Linie modellierte (erwartete) Mortalität ohne Influenza; gestrichelte, blaue Linie oberer Vertrauensbereich; senkrechte, gepunktete Linien Jahreswechsel. (Mod. nach Ansart et al.; [22]), Abbildung mit freundlicher Genehmigung @ John Wiley and Sons.

Einfluss des Weltkriegs auf die Mortalität wurden die Jahre 1915-1917 ausgeschlossen. In einem linearen Regressionsmodell über alle Länder wurde zusätzlich untersucht, welchen Einfluss die Faktoren Pro-Kopf-Einkommen und Breitengrad hatten. Dabei ergab sich, dass die Variable Breitengrad vernachlässigbar war, dass aber das historische, damalige Pro-Kopf-Einkommen fast zur Hälfte die sehr große Variation der pandemischen Mortalität „erklärte“. Zum Beispiel war die Exzess-Mortalität einzelner indischer Provinzen (6000-8000 pro 100.000$)$ ca. 20 -fach höher als in Australien (300/100.000). Somit waren die damaligen Auswirkungen der Pandemie nicht nur von dem Erreger und seinen Eigenschaften, sondern zu einem großen Teil auch von der damaligen wirtschaftlichen Situation in den Ländern abhängig. Die für Deutschland geschätzte, historische Mortalität wurde von Murray mit 760/100.000 angegeben, was bei der damaligen Bevölkerung einer Todesfallzahl von etwa 470.000 entspricht, also etwa das doppelte der Schätzungen von Johnson u. Mueller (•Tab. 1).

Kurz vor Ausbruch der Pandemie 2009 veröffentlichten Ansart et al. die Ergebnisse ihrer Schätzungen der pande- mischen Mortalität 1918/1919 in 14 Ländern Europas [22]. Grundlage waren historische, monatliche Gesamtsterbefalldaten, die im Jahr 1954 vom Pariser Nationalen Institut für Demographische Studien für den Zeitraum 1906-1936 auf die gleiche Weise für 14 europäische Länder zusammengetragen worden waren. Dies eröffnete die Möglichkeit, ExzessMortalitäts-Schätzungen durchzuführen und dabei saisonale Schwankungen mit einzubeziehen. Nach Ausschluss der Quartale, in denen sich Influenzaepidemien ereigneten, wurde mittels eines periodischen Regressionsmodells und unter Verwendung der Jahre 1906-1917 eine Basislinie (entsprechend einer Hintergrundmortalität) erstellt, die darauf in die Jahre 1918-1922 extrapoliert wurde. Ein Exzess (ohne Influenza im Hintergrund) wurde dann berechnet, wenn in zwei aufeinander folgenden Monaten die monatliche, beobachtete Mortalität oberhalb der oberen Grenze des erwarteten Prädiktionsintervalls lag, welches definiert war als der Punktschätzer plus das 1,96-Fache der Standardabweichung. Wegen der während der Jahre 1914-1918 beobachteten stufenförmig erhöhten Gesamtmortalität in Deutschland wurden für den linearen Trend die Jahre 1906-1913 und 1919-1936 verwendet. Deskriptiv zeigten Ansart et al. in Deutschland eine frühe Welle von März bis Juli 1918 sowie eine zweite Welle von Oktober bis Dezember 1918 (• Abb. 2). Aus diesen beiden Wellen wurde eine Exzess-Mortalität von 691/100.000 und eine pandemiebedingte Todesfallzahl von 426.574 geschätzt (gerundet 426.600; • Tab. 1). Eine dritte Welle im Frühjahr 1919 war in Deutschland - anders als in einigen anderen europäischen Ländern - nicht zu beobachten.

Eigene Berechnungen: Die jährliche Mortalität einer Auswahl registrierter Todesfälle sowie der Gesamtzahl aller Todesfälle für den Zeitraum von 1892-1971 zeigt, dass während der pandemischen Phase 1918/1919 eine klare Exzess-Mortalität bei den Gesamttodesfällen v. a. für das Jahr 1918 zu erkennen ist, für die Pandemien 1957/1958 und 1968-1970 aber höchstens andeutungsweise (• Abb. 3). Die Mortalitätskurven für die drei Todesursachen Tuberkulose, Pneumonie und Altersschwäche zeigen zwar einen Gipfel um das Jahr 1918, bei näherer Betrachtung sieht man jedoch, dass ein isolierter Ein-Jahres-Gipfel in 1918 nur für Pneumonien zu sehen ist, während es bei Altersschwäche und Tuberkulose schon im Jahr $1917 \mathrm{zu}$ einem deutlichen Anstieg gekommen war. Die Zahl der „offiziellen“ Influenzatodesfälle stieg von $6799 \mathrm{im} \mathrm{Jahr} 1917$ auf 185.815 im Jahr 1918 (E. Michels, persönliche Kommunikation). Die Exzess-Schätzung unter Verwendung der Gesamttodesfallzahlen 1917 und 1919 ergab eine ExzessTodesfall-Schätzung (mit Influenza im Hintergrund) von 442.300 (Mortalität 716/100.000).

Bewertung: Am vertrauenswürdigsten erscheinen die Schätzungen von Ansart et al. (gerundet 426.000 Todesfälle, Mortalität von 691/100.000), da diese auf dem detailliertesten Datenmaterial aufbauen und eine gut begründete und validierte Analysemethode verwenden. Die Ergebnisse zeigen eine zeitliche und quantitative Vergleichbarkeit mit denen aus anderen europäischen Ländern und erhalten dadurch zusätzliche Glaubwürdigkeit. 


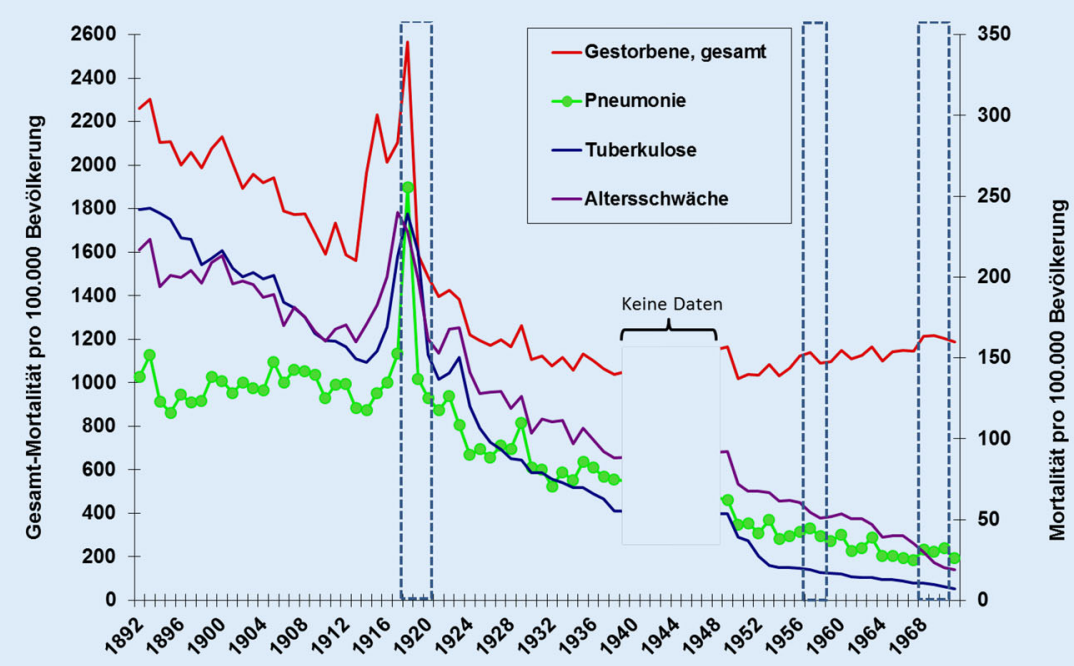

Abb. $3 \Delta$ Jährliche Mortalität pro 100.000 Bevölkerung für Gesamtsterbefälle (linke y-Achse) sowie die Todesursachendiagnosen Tuberkulose, Pneumonie und Altersschwäche (rechtey-Achse). Die drei umrahmten Felder kennzeichnen die pandemischen Phasen 1918-1920, 1957/58 und 1968-1970. Zwischen 1939 und 1948 waren keine Daten verfügbar

\section{Pandemie 1957/1958}

Identifizierte Literatur: Für die Pandemie 1957/1958 wurden zwei Arbeiten identifiziert. Meyer u. Rückert verwendeten Sterbetafeln (aller Todesfälle unabhängig von der Ursache) der damaligen Bundesrepublik Deutschland (BRD) als Grundlage und führte Zeitreihenanalysen durch [24]. Dabei konnten nach Subtraktion des Trends, der saisonalen Schwankungen und der Entwicklungen über mehrere Jahre hinweg Anomalien (Exzess-Sterblichkeiten; mit Influenza im Hintergrund) identifiziert werden. Aufgrund der Gleichzeitigkeit einer Erhöhung der Sterbezahlen bei Grippe wurden einige dieser Anomalien Grippemonaten zugeschrieben, das Wort „Pandemie“ fällt jedoch nicht. Für den Oktober 1957 wurden so 13.000-15.000 „Sterbefälle auf das Ereignis Grippewelle" zurückgeführt [24], extrapoliert auf die BRD und das damalige Gebiet der Deutschen Demokratischen Republik (DDR), ergeben sich 17.200-19.800 Todesfälle (gemittelte Mortalität 26/100.000; ه Tab. 1). Die zweite Veröffentlichung [13] war die Grundlage für die (eigene) Umrechnung der Mortalität in die Zahl der pandemiebedingten Exzess-Todesfälle (s. nächster Absatz).
Eigene Berechnungen: Für die Pandemie durch A(H2N2) 1957 ergab sich für die Monate Oktober und November 1957, nach Umrechnung der Exzess-Mortalität (ohne Influenza im Hintergrund), eine Zahl von (gerundet) 29.100 Todesfällen im Gebiet der damaligen BRD und DDR (Mortalität: 40/100.000).

Bewertung: Die eigenen Berechnungen erscheinen v. a. wegen der detaillierteren methodischen Dokumentation der zugrunde liegenden Veröffentlichung als glaubwürdigster Ansatz für die Schätzung der pandemischen Exzess-Todesfälle 1957/1958 (gerundet 29.100 Todesfälle, Mortalität 40/100.000).

\section{Pandemie 1968-1970}

Identifizierte Literatur: Für die Pandemie ab 1968 wurden drei Veröffentlichungen gefunden. Die erste ist wiederum die Arbeit von Meyer, der für die Monate Dezember 1969 und Januar 1970 in der damaligen BRD einen Exzess (mit Influenza im Hintergrund) von 40.000 pandemiebedingten Todesfällen berechnete (entsprechend einer Mortalität von 66/100.000; • Tab. 1). Unter Annahme einer identischen Mortalität auch in der DDR wären in den beiden Teilen Deutschlands 51.200 Personen infolge der Pandemie gestorben. Als weitere Quelle wurde für die Pandemie 1968-1970 das Kapitel zu „Grippe“ aus dem Buch Mortalität und Todesursachen identifiziert [25]. In diesem Buch wird die Mortalität aufgrund verschiedener Todesursachen, z. B. des Herz-KreislaufSystems, des Atmungssystems und anderer Krankheitsgruppen thematisiert. Obwohl dort angesprochen wird, dass sich Grippeepidemien in der Todesursachenstatistik nicht nur bei Grippe, sondern auch bei anderen Krankheiten der Atmungsorgane niederschlugen, wurden für die hier interessierenden Jahre der Pandemien 1957/1958 bzw. 1968-1970 keine Berechnungen durchgeführt. Allerdings fanden sich im Anhang Sterbetafeln zur Todesursache „Grippe“, getrennt für Männer und Frauen und getrennt für die damalige Bundesrepublik bzw. die DDR. Addiert man (registrierend) für die Pandemie 1968-1970 die dortigen Angaben in den vier Strata, ergibt sich eine Summe von 15.314 (gerundet 15.300) Todesfällen mit Ursache Grippe (Mortalität 20/100.000). Die dritte Veröffentlichung [13] ist wiederum Grundlage für die (eigene) Umrechnung der Mortalität in die Zahl der pandemiebedingten ExzessTodesfälle (s. nächster Absatz).

Eigene Berechnungen: Für die A(H3N2)-Pandemie ab 1968 wurde die Exzess-Mortalität (ohne Influenza im Hintergrund) der Monate Dezember 1969 und Januar 1970 herangezogen, welche in der Publikation als die ersten Monate der Pandemie angegeben sind [13]. Die Zahl der pandemiebedingten Todesfälle betrug in der BRD und DDR (gerundet) 46.900 (Mortalität: 60/100.000; s. - Tab. 1).

Bewertung: Die eigenen Berechnungen erscheinen v. a. wegen der detaillierteren methodischen Dokumentation als glaubwürdigster Ansatz für die Schätzung der pandemischen Exzess-Todesfälle 1968-1970 (gerundet 46.900 Todesfälle, Mortalität 60/100.000).

\section{Pandemie 2009}

Identifizierte Literatur: Neben drei publizierten Arbeiten, die von den Autoren dieser Arbeit (mit-)verfasst wurden, konnten keine weiteren relevanten Ver- 
öffentlichungen identifiziert werden. Da die WHO die Pandemie am 10.08.2010 als beendet erklärt hatte, wurde für die Auswirkung der Pandemie 2009 in Deutschland nur die Welle von Oktober 2009 bis Januar 2010 berücksichtigt, nicht jedoch die ohnehin relativ schwache Influenzawelle 2010/2011.

Von Wilking et al. erfolgte eine Auswertung der nach Infektionsschutzgesetz und der Verordnung über die Meldepflicht bei Influenza, die durch das erstmals im April 2009 in Nordamerika aufgetretene neue Virus („Schweine-Grippe") hervorgerufen wird, an das Robert Koch-Institut gemeldeten laborbestätigten Influenzafälle, die nach Einschätzung des übermittelnden Gesundheitsamts aufgrund der Erkrankung durch das pandemische Virus A(H1N1)pdm09 verstarben (registrierende Methode) [14]. Diese ergab eine Anzahl von 252 (gerundet 250) Personen und entspricht einer Mortalität von 0,31 pro 100.000 Einwohner (- Tab. 1). Der Altersmedian der Verstorbenen lag bei 47 Jahren, mit einem Interquartilsbereich von 29-57 Jahren.

Eine weitere Veröffentlichung beschreibt ein großes internationales Projekt unter dem Akronym The Global Pandemic Mortality Burden project (GLaMOR), welches sich zum Ziel gemacht hatte, die globale Exzess-Mortalität der Pandemie 2009 zu schätzen [8]. Deutschland war dabei eines von 20 Ländern, bei denen eine datenbasierte Exzess-Schätzung (ohne Influenza im Hintergrund) vorgenommen wurde. Die verwendete Methode ist in der Veröffentlichung beschrieben [8], jedoch wurden dort nicht die Ergebnisse für Deutschland veröffentlicht, wo sich eine kleine, aber signifikante Anzahl an Exzess-Todesfällen in der Altersgruppe der unter 65-Jährigen ergeben hatte. Wenn alle Sterbefälle unabhängig von der Todesursache berücksichtigt wurden, wurden 348 (gerundet 350; $95 \%$-Konfidenzintervall, KI, 50-646) Exzess-Todesfälle geschätzt. Bei den respiratorischen oder kardiovaskulären Todesfällen wurden 223 (95\%-KI, 71-375), bei den respiratorischen Todesfällen 152 (95\%-KI, 97-202) und bei den Todesfällen, die mit „Grippe oder Pneumonie“ kodiert waren, 108 (95\%-KI, 83-133) Exzess-
Todesfälle geschätzt. Dabei sind die als „Grippe oder Pneumonie“ kodierten Todesfälle eine Teilmenge der respiratorischen Todesfälle und diese wiederum der respiratorischen oder kardiovaskulären Todesfälle. Bei den respiratorischen Todesfällen konnte etwa die Hälfte auf einen Exzess in der Altersgruppe der 45bis 64-Jährigen zurückgeführt werden. Dies passt gut zu den Ergebnissen der Meldedatenauswertung (s. die Arbeit von Wilking et al. [14]).

In der dritten Veröffentlichung wurde das Schätzverfahren nach Zucs et al. [1] verwendet und ergab keine ExzessMortalität für die Pandemie 2009 [15].

Bewertung: Die Todesfallzahl der ersten Arbeit erscheint als absoluter Minimalwert, da die über das Meldegesetz übermittelten Todesfälle eine falsch-positive Registrierung quasi ausschließen. Die zweite Arbeit stimmt bezüglich der Altersbetonung der mittelalten Erwachsenen mit dem Ergebnis aus der registrierenden Methode von Wilking et al. [14] gut überein. Wegen der bewährten, verwendeten modellierenden Schätzungen kommt das schätzende Verfahren [8] zu vermutlich noch plausibleren Ergebnissen. Die Datengrundlage der dritten Arbeit hat eine zu grobe zeitliche Auflösung und wird daher hinter den ersten beiden Arbeiten eingestuft.

\section{Synopse der Todesfall- und Mortalitätsdaten aus der Literatur und eigenen Berechnungen}

Die synoptische Darstellung der Todesfallzahlen bzw. Mortalitäten aus allen genannten Quellen ist in • Abb. 4 wiedergegeben. Der Unterschied in der Mortalität zwischen den Extremen 1918 und 2009 beträgt bei den als am vertrauenswürdigsten eingeschätzten Schätzungen 691 bzw. 0,43 pro 100.000 Bevölkerung. Die der Influenza zugeschriebene ExzessMortalität ist in 1918 mehr als 1000 fach höher als in 2009.

\section{Vergleich der Letalität in Deutsch- land mit globalen Schätzungen der Weltgesundheitsorganisation}

Die geschätzte Letalität der Pandemien betrug in Deutschland 1918/19192,76 \%,
$1957 / 19580,13 \%, 1968-19700,29 \%$ und $20090,0048 \%$. Auf der Originalskala und bei logarithmisch transformierten Werten ergaben sich beim Vergleich mit den Zahlen der WHO nur geringe Unterschiede: Die Letalität in Deutschland war während der Pandemie 1918/1919 höher als weltweit geschätzt, in den mittleren Pandemien sehr ähnlich und in der jüngsten Pandemie 2009 etwas niedriger (- Abb. 5). In - Tab. 2 finden sich alle Kennzahlen für Deutschland auf einen Blick.

\section{Diskussion}

Wir präsentieren eine Zusammenstellung der Ergebnisse einer Literaturrecherche, ergänzt um eigene Berechnungen zur Auswirkung der drei Pandemien des letzten Jahrhunderts sowie der Pandemie 2009 im Hinblick auf die Gesamtmortalität und die geschätzte Zahl der in Deutschland verstorbenen Personen. Nach Bewertung der verwendeten Daten und Methoden wurde der aus Sicht der Autoren für jede Pandemie vertrauenswürdigste Wert ausgewählt.

Für die Pandemie 1918/1919 liegen von einer Reihe anderer Länder Schätzungen zur Exzess-Mortalität vor. Die für Deutschland vertrauenswürdigste betrug 691/100.000 [22]. In den USA wurde z. B. eine Exzess-Mortalität zwischen 280 pro 100.000 Einwohner [26] und bis zu 650 pro 100.000 Einwohner [27] geschätzt, in Großbritannien von 590 pro 100.000 [26] und in Gesamteuropa von 1100 pro 100.000 [22] mit einem Nord-Süd-Gradienten, i. e. einer höheren Mortalität in den südlichen Ländern Europas. Besonders schwer schienen asiatische Länder betroffen gewesen zu sein, mit 1800 pro 100.000 Exzess-Mortalität in Singapur [28] und bis $\mathrm{zu} 4400$ pro 100.000 in Indien [21]. Die geographischen Unterschiede der Exzess-Mortalitätsraten 1918 waren immens. Sicherlich hat auch die finale Phase des Kriegs bei einigen Ländern die pandemische Mortalität mit beeinflusst, besonders augenscheinlich ist aber die Assoziation von niedrigem Pro-Kopf-Einkommen und hoher Mortalität [21]. Diese spiegelt vermutlich zu großen Teilen den Einfluss von Faktoren wider, die sich indirekt auf die 


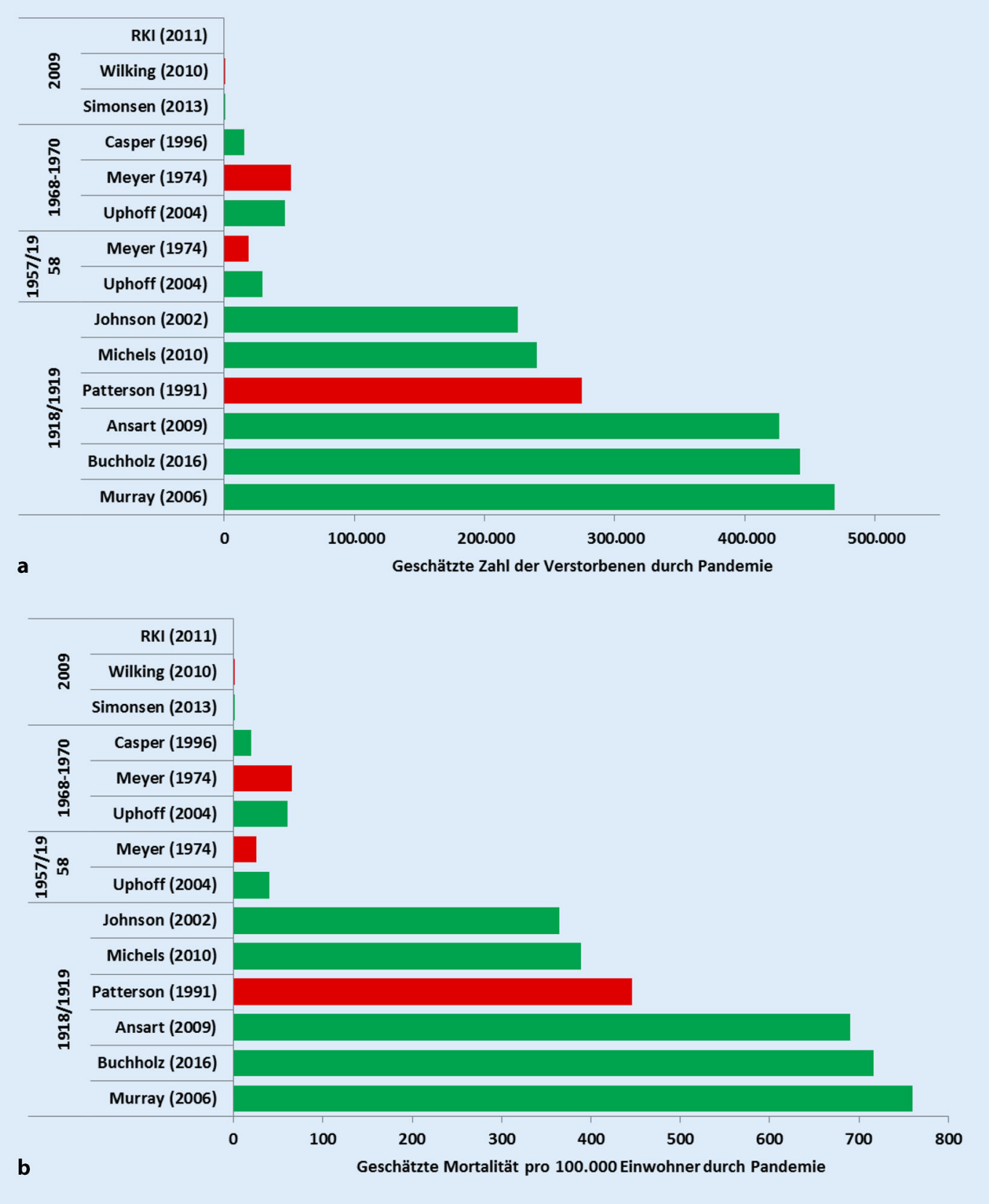

Abb. $4 \varangle$ Aufgrund von Exzess-Todesfall-Schätzungen oder Auswertungen von Meldedaten ermittelte Zahl der Todesfälle (oben) bzw. Mortalität (unten) aufgrund der Influenzapandemien 1918/1919, 1957/1958, 1968-1970 und 2009. Angegeben sind die Erstautoren der Veröffentlichungen und die Jahreszahl der Veröffentlichung. Rote Balken stellen die von den Autoren vorgeschlagenen, vertrauenswürdigsten Schätzwerte für die jeweiligen Pandemien dar. Die Autoren bzw. Auswertungen beziehen sich auf 0 Tab. 1

Mortalität auswirken können. In Frage kommen u. a. der Ernährungsstatus der Bevölkerung, Begleiterkrankungen oder die Kapazität für unterstützende, lebenserhaltende Behandlungsmöglichkeiten [21].

Für die Pandemien 1957/1958 und 1968-1970, für die eine monatliche Zeitreihe zur Verfügung stand, wurde von Uphoff eine mathematische, modellierende Methode angewendet, um die Zahl der Exzess-Todesfälle zu schätzen. Die Methodik unterscheidet sich zwar von derjenigen, die Ansart et al. angewendet hatten, jedoch folgt sie einem ähnlichen Prinzip (Etablierung einer Basislinie ohne Influenza; Extrapolierung in die Zeitperioden mit Influenza; Differenzbildung der aufgetretenen (beobachteten) Zahl der Todesfälle minus Zahl der zu erwartenden Zahl der Todesfälle ohne Pandemie, repräsentiert durch die Basislinie), und wurde als Methodik veröffentlicht [1]. Dabei ergaben sich für 1957/1958 eine gerundete Zahl von 29.100 Pandemietodesfällen (Exzess-Mortalität 40/100.000; stärkere Welle im September/Oktober
1957, schwächere Nachwelle im Januar/ Februar 1958) und für 1968-1970 eine gerundete Zahl von 46.900 Pandemietodesfällen (Exzess-Mortalität 60/100.000; eine Hauptwelle im Dezember 1969 und Januar 1970). Obwohl die ersten pandemischen A(H3N2)-Viren zu Beginn des Jahres 1968 in Hong Kong entdeckt wurden [29], dauerte es bis zum Dezember 1968, bis sich das Virus in den USA großflächig verbreitet hatte. In Deutschland könnte es im Frühjahr 1969 eine leichte Vor- bzw. erste Welle gegeben haben; diese ist aber nur durch 


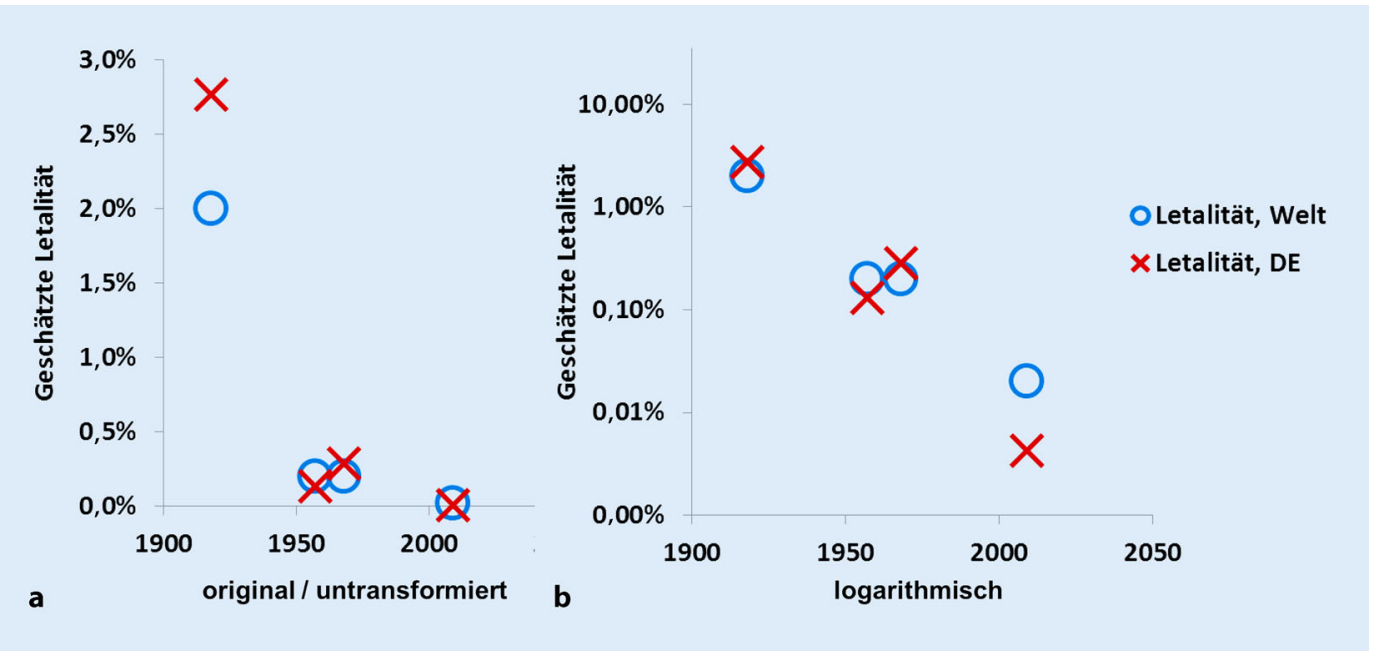

Abb. 5 \ Vergleich der von der Weltgesundheitsorganisation geschätzten, weltweiten Letalität für die vier Pandemien 1918/1919, 1957/1958, 1968-1970 und 2009 und der aufgrund dieser Arbeit geschätzten Letalität in Deutschland. Links: nicht transformierte Zahlen (Originalskala), rechts: logarithmische Auftragung. Für die Berechnung der Letalität wurden die von den Autoren dieser Arbeit als am vertrauenswürdigsten bewerteten Zahlen der pandemiebedingten Todesfälle verwendet

eine Literaturstelle aus der damaligen DDR, nicht jedoch aus der damaligen BRD belegt [13]. Die eigentliche pandemische Welle traf Deutschland erst im Dezember 1969/Januar 1970. Interessant ist, dass zumindest für Deutschland die Pandemie 1957 etwas schwächer ausfiel als diejenige in 1968/1969, während die aus der Literatur entnommenen Schätzungen einzelner Länder eher eine größere Betroffenheit bei der Pandemie 1957 angeben (USA: Exzess-Mortalität 1957: 39/100.000, vs. 1968: 14/100.000 [30], Singapur: Exzess-Mortalität 1957: 47/100.000, vs. 1968: 27/100.000 [28]).

Bei der Eruierung der Zahl der Todesfälle bzw. Mortalität aufgrund der Pandemie 2009 standen (im Gegensatz zu den Pandemien 1918/1919, 1957/1958 und 1968-1970) bei den Meldedaten [14] sowie bei der Modellierung im Rahmen des Projekts GLaMOR [8] altersbezogene Todesfalldaten zur Verfügung. Da der Interquartilsbereich des Alters der gemeldeten Todesfälle bei 29-57 Jahren lag, war die im Rahmen des Vorgehens bei GLaMOR durchgeführte Stratifizierung nach „,unter 65 Jahren“ bzw. „65 Jahre und älter" sinnvoll. Durch die Trennung wird in der jüngeren Altersgruppe (mit relativ niedriger Hintergrundmortalität) der Großteil der pandemiebedingten Verstorbenen erkennbar, während ein kleinerer Teil in der älteren Altersgruppe (mit hoher Hintergrundmortalität) ,untergeht“. In der Tat ermöglichte nur die getrennte Betrachtung dieser Altersgruppen die Erkennung der Exzess-Todesfälle in der jüngeren Altersgruppe. In einem Gesamtmodell (ohne Stratifizierung) war die Zahl der Exzess-Todesfälle in der jüngeren Altersgruppe nicht mehr erkennbar, weil die schiere Zahl der Gesamttodesfälle in der älteren Altersgruppe (in den Monaten November und Dezember 2009 starben unter den mindestens 65 Jahre alten Personen ca. 125.000) dominierte und die geringen Abweichungen in der jüngeren Altersgruppe völlig maskierte. Dies dürfte auch der maßgebliche Grund sein, warum in der dritten Arbeit [15] keine Exzess-Mortalität erkennbar war.

Die von Wilking et al. [14] genannten bzw. im Rahmen der Mitarbeit bei Simonsen [8] berechneten Zahlen gehen, auch bei Berücksichtigung des $95 \%$ KI, nicht über 1000 Todesfälle hinaus. Die 252 (gerundet 250) Todesfälle, die im Rahmen des Meldesystems erhoben und verifiziert wurden, sind im Grunde "handverlesen“ und als Minimum anzunehmen. Die modellierende Zeitreihenanalyse fußt wiederum auf dem Konzept der Exzess-Todesfallberechnung; die zeitliche Auflösung der verwendeten Daten (wöchentliche Aggregation des Todesdatums) ist hoch. Da die Datengrund- lage das Jahr 2010 nicht einbezog, könnte die errechnete Anzahl von 348 (gerundet 350) Todesfällen zu niedrig sein und die eigentliche Zahl noch um einen Faktor von bis zu $30 \%$ [8] höher liegen als angegeben. Global wurde, wie schon Murray et al. auch für die Schätzung für 1918 beschrieben haben, eine ausgeprägte regionale Heterogenität beobachtet, mit einer bis zu 20-fach höheren Mortalität in einigen Ländern der amerikanischen Kontinente im Vergleich zu europäischen Ländern (Exzess-Mortalität in Deutschland: 0,4/100.000, Frankreich 0,3/100.000; China: 2,1/100.000, Mexiko: 5,2/100.000) [8]. Zusätzlich bedeutsam war, dass global - je nach gewählter Datengrundlage und Schätzmethode zwischen $62 \%$ und $85 \%$ der Todesfälle bei Personen auftraten, die jünger als 65 Jahre alt waren [8].

Die als am vertrauenswürdigsten bewerteten Berechnungen der vier Pandemien haben gemeinsam, dass bei allen vieren eine Exzess-Mortalitäts-Schätzung erfolgte. Darüber hinaus wurde durchgehend ein Verfahren gewählt, bei dem Influenzawellen nicht in den Hintergrunddaten enthalten waren. Dadurch ist die Methodik dieser vier Schätzwerte relativ homogen.

Für eine weitere Validierung der von uns identifizierten, plausibelsten Werte wurden aus den Todesfallzahlen und 
Tab. 2 Synopse aller vorgeschlagenen Kennzahlen der Pandemien 1918/1919, 1957/1958,

1968-1970 und 2009; Deutschland

\begin{tabular}{|c|c|c|c|c|c|}
\hline Pandemie & Bevölkerung $^{a}$ & $\begin{array}{l}\text { Erkrankungsrate } \\
(\%)\end{array}$ & Todesfälle $^{c}$ & $\begin{array}{l}\text { Mortalität } \\
(/ 100.000)^{c}\end{array}$ & Letalität (\%) \\
\hline 1918/1919 & 61.756 .930 & 25 & 426.600 & 691 & 2,76 \\
\hline 1957/1958 & 72.030 .866 & 31 & 29.100 & 40 & 0,13 \\
\hline 1968-1970 & 78.069 .482 & 21 & 46.900 & 60 & 0,29 \\
\hline 2009 & 81.802 .257 & 9 & 350 & 0,43 & 0,0048 \\
\hline
\end{tabular}

a'Die Bevölkerung umfasst 1957/1958 und 1968-1970 sowohl die alte Bundesrepublik wie auch die DDR

bErkrankungsraten für 1918/1919, 1957/1958 und 1968-1970 übernommen aus Gani (EID, 2005); für 2009 geschätzt aus Daten der Arbeitsgemeinschaft Influenza und von GrippeWeb (Arbeitsgemeinschaft Influenza, Saisonbericht 2009/10 und 2010/11)

'Die von den Autoren in dieser Arbeit als am vertrauenswürdigsten bewertete Schätzer

unter Einbezug von Annahmen zu den Erkrankungsraten Letalitäten berechnet, die dann mit globalen Werten der WHO verglichen werden konnten. Die Übereinstimmung war insgesamt zufriedenstellend. Die etwas höhere Letalität 1918/1919 könnte mit der sehr schlechten Gesamtsituation der Bevölkerung zu Ende des 1. Weltkriegs zusammenhängen; dagegen war die geschätzte Letalität 2009 (nur im logarithmischen Maßstab erkennbar) etwas niedriger als weltweit. Allerdings sind die für Deutschland geschätzten Letalitäten stark abhängig von den getroffenen Annahmen, z. B. dem Anteil der Influenzakranken, die 2009 einen Arzt aufsuchten (40\%). Ein niedrigerer Anteil würde zu einer noch niedrigeren Letalität führen. Eine in Deutschland im Vergleich zur Weltbevölkerung niedrigere Letalität könnte viele Ursachen haben, u. a. die gute medizinische Versorgung.

Insgesamt ist auffallend, dass die geschätzten pandemieassoziierten Mortalitäten in den vier letzten Pandemien sehr stark voneinander abweichen. Neben den schon genannten „äußeren Bedingungen“, wie z. B. die über die Jahre verbesserte Gesundheitsversorgung mit der erst nach 1918 bestehenden Möglichkeit, bakteriell bedingte sekundäre Pneumonien mit Antibiotika spezifisch zu therapieren sowie einer deutlich besseren gesundheitlichen Allgemeinsituation der Bevölkerung, sind sicherlich auch erregerspezifische Eigenschaften für die unterschiedliche Mortalität mit verantwortlich.

Zusammenfassend kann gesagt werden, dass - ausgehend von einer Sichtung und kritischen Bewertung der Literatur sowie eigenen Schätzungen bzw. Auswertungen - für jede der vier vergangenen Influenzapandemien - ein vertrauenswürdiger Schätzwert für Deutschland vorgeschlagen werden konnte. Die resultierende Letalität entspricht den von der WHO angegebenen globalen Werten. Alle vorgeschlagenen Werte stellen Schätzungen dar, die mittels einer Exzess-Todesfallberechnung - jeweils ohne Influenza im Hintergrund -, aufbauend auf monatlichen oder wöchentlichen Zeitreihen, ermittelt wurden. Die extreme Heterogenität der Schätzwerte repräsentiert vermutlich ebenso die Erregervarianz wie andere Faktoren, z. B. das Pro-Kopf-Einkommen, das Gesundheitssystem, die therapeutischen Möglichkeiten, die Bevölkerungsimmunität etc., und zeigt auf, dass die Auswirkungen einer zukünftigen Pandemie kaum vorhergesagt werden können.

\section{Korrespondenzadresse}

\section{Dr. U. Buchholz}

Abteilung für Infektionsepidemiologie, Robert Koch-Institut

Seestraße 10, 13353 Berlin, Deutschland buchholzu@rki.de

Danksagung. Unser Dank geht an Yvonne Bichel für Ihre Unterstützung bei der Literaturrecherche, an Eckard Michels für die Zurverfügungstellung einzelner Daten sowie die Gutachter für das wiederholte sorgfältige Lesen des Manuskripts und ihre vielfachen, wertvollen Vorschläge.

\section{Einhaltung ethischer Richtlinien}

Interessenkonflikt. U. Buchholz, S. Buda, A. Reuß, W. Haas und H. Uphoff geben an, dass kein Interessenkonflikt besteht.

Dieser Beitrag beinhaltet keine von den Autoren durchgeführten Studien an Menschen oder Tieren.

\section{Literatur}

1. Zucs P, Buchholz U, Haas W, Uphoff H (2005) Influenza associated excess mortality in Germany. Emerg Themes Epidemiol 2(6):1985-2001

2. Schelhase T, Weber S (2007) Mortality statistics in Germany. Problems and perspectives. Bundesgesundheitsblatt Gesundheitsforschung Gesundheitsschutz 50:969-976

3. World Health Organization (2009) Comparing deaths from pandemic and seasonal influenza. http://www.who.int/csr/disease/swineflu/notes/ briefing_20091222/en/. Zugegriffen: 23. Febr. 2015

4. Serfling RE, Sherman IL, Houseworth WJ (1967) Excess pneumonia-influenza mortality by age and sex in three major influenza A2 epidemics, United States, 1957-58, 1960 and 1963. Am J Epidemiol 86:433-441

5. Reichert TA, Sugaya N, Fedson DS et al (2001) The Japaneseexperiencewith vaccinating schoolchildren against influenza. N Engl J Med 344:889-896

6. Pitman RJ, Melegaro A, Gelb D et al (2007) Assessing the burden of influenza and other respiratory infections in England and Wales. J Infect 54:530-538

7. Thompson WW, Shay DK, Weintraub E et al (2003) Mortality associated with influenza and respiratory syncytial virus in the United States. JAMA 289:179-186

8. Simonsen L, Spreeuwenberg P, Lustig Ret al (2013) Global mortality estimates for the 2009 Influenza Pandemic from the GLaMOR project: a modeling study. PLOS Med 10:e1001558

9. Jordan EO (1927) Epidemic influenza: a survey. American Medical Association, Chicago

10. Johnson N, Mueller J (2002) Updating the accounts: global mortality of the 1918-1920 "Spanish" influenza pandemic. Bull Hist Med 76(1):105-115

11. World Health Organization (2015) WHO pande mic influenza risk management WHO interim guidance. http://www.who.int/influenza/ preparedness/pandemic/GIP_Pandemic InfluenzaRiskManagementInterimGuidance Jun2013.pdf(Erstellt:).Zugegriffen: 19. März 2015

12. Dawood FS, luliano AD, Reed C et al (2012) Estimated global mortality associated with the first 12 months of 2009 pandemic influenza A H1N1 virus circulation: a modelling study. Lancet Infect Dis 12:687-695

13. UphoffH,Stilianakis NI (2004) Influenza-associated excess mortality from monthly total mortality data for Germany from 1947 to 2000 . Methods Inf Med 43:486-492

14. Wilking $H$, Buda S, Von Der Lippe E et al (2010) Mortality of 2009 pandemic influenza $A(H 1 N 1)$ in Germany. Euro Surveill. pii:19741

15. Robert Koch-Institut (2011) Schätzung der Influenza-bedingten Todesfälle während der Saison 2008/09 und der pandemischen Saison 2009/10. Epidemiol Bull 10:75-77 (http://edoc. 


\section{Originalien und Übersichten}

rki.de/documents/rki_fv/rekpWs881mymw/PDF/ 29reJdleoV1c.pdf; Zugegriffen: 19.03.2015)

16. Gani R (2005) Potential impact of antiviral drug use during influenza pandemic. Emerg Infect Dis 11:1355-1362

17. Arbeitsgemeinschaft Influenza (2015) Saisonbericht 2009/10. www.influenza.rki.de/agi > Saisonberichte (Erstellt: ). Zugegriffen: 12. Aug. 2015

18. Arbeitsgemeinschaft Influenza Jahresbericht 2010/11. www.influenza.rki.de/agi > Saisonberichte.Zugegriffen: 12. Aug. 2015

19. Patterson KD, Pyle GF (1991) The geography and mortality of the 1918 influenza pandemic. Bull Hist Med 65(1):4-21

20. Michels E (2010) Die "Spanische Grippe" 1918/19. Verlauf, Folgen und Deutungen in Deutschland im Kontext des Ersten Weltkriegs. Vierteljahrsh Zeitgesch 58:1-33

21. Murray CJ, Lopez AD, Chin B, Feehan D, Hill KH (2006) Estimation of potential global pandemic influenza mortality on the basis of vital registry data from the 1918-20 pandemic: a quantitative analysis. Lancet 368:2211-2218

22. Ansart S, Pelat C, Boelle PY et al (2009) Mortality burden of the 1918-1919 influenza pandemic in Europe. Influenza Other Respir Viruses 3:99-106

23. Science at Home (2015) Die Opfer des 1. Weltkriegs. http://www.science-at-home.de/ wiki/index.php/Die_Opfer_des_1._Weltkriegs. Zugegriffen: 2. Juni 2015

24. Meyer K, Rückert GR (1974) Allgemeine Sterbetafel 1970/72. Wirtsch Stat 7:465-475

25. Casper W, Wiesner G, Bergmann KE, Robert Koch-Institut (1996) Kapitel 8 („Krankheiten des Atmungssystems“), Unterkapitel 8.1 ("Grippe (ICD 9:487)“). In: Mortalität und Todesursachen. Robert Koch-Institut, Berlin, S318-332

26. Richard SA, Sugaya N, Simonsen L et al (2009) A comparative study of the 1918-1920 influenza pandemic in Japan, USA and UK: mortality impact and implications for pandemic planning. Epidemiol Infect 137:1062-1072

27. Glezen WP (1996) Emerging infections: pandemic influenza. Epidemiol Rev 18:64-76

28. Lee VJ, Chen MI, Chan SP et al (2007) Influenza pandemics in Singapore, a tropical, globally connected city. Emerg Infect Dis 13:1052-1057

29. Anonymous US Department of health and human services. Pandemie flu history. http://www.flu. gov/pandemic/history/. Zugegriffen: 30. Sept. 2014

30. Simonsen L, Clarke MJ, Schonberger LB et al (1998) Pandemic versus epidemic influenza mortality: a pattern of changing age distribution. J Infect Dis 178:53-60 